\title{
Numerical Study of Enhanced Performance in InGaN Light-Emitting Diodes with Graded-composition AlGaInN Baniers
}

\author{
Su Jin Kim and Tae Geun Kim* \\ School of Electronics and Electrical Engineering, Korea University, Seoul 136-701, Korea
}

(Received October 30, 2012 : revised December 26, 2012 : accepted January 3, 2013)

\begin{abstract}
In this paper, we report the effect of $\mathrm{GaN} /$ graded-composition $\mathrm{AlGaInN/GaN} \mathrm{quantum} \mathrm{barriers} \mathrm{in} \mathrm{active}$ regions on the electrical and optical properties of GaN-based vertical light emitting diodes (VLEDs). By modifying the aluminum composition profile within the AlGaInN quantum barrier, we have achieved improvements in the output power and the internal quantum efficiency (IQE) as compared to VLEDs using conventional $\mathrm{GaN}$ barriers. The forward voltages at $350 \mathrm{~mA}$ were calculated to be 3.5 and $4.0 \mathrm{~V}$ for VLEDs with $\mathrm{GaN} /$ graded-composition $\mathrm{AlGaInN} / \mathrm{GaN}$ barriers and $\mathrm{GaN}$ barriers, respectively. The light-output power and IQE of VLEDs with GaN/graded-composition AlGaInN/GaN barriers were also increased by $4.3 \%$ and $9.51 \%$, respectively, as compared to those with $\mathrm{GaN}$ barriers.
\end{abstract}

Keywords: Light-emitting diodes (LEDs), AlGaInN, Quantum barriers, Numerical simulation OCIS codes : (230.3670) Light-emitting diodes; (230.0230) Optical devices

\section{INTRODUCTION}

InGaN/GaN-based light-emitting diodes (LEDs) have attracted considerable interest because of their various industrial application in general lighting, backlight units in liquid crystal displays (LCD), full-color displays, and automotive head lamps [1-7]. Nowadays, the application of GaN LEDs has been extended to general lighting, which requires high-brightness and high-power operation. For high-brightness and high-power applications of solid-state general lighting, LEDs have to be operated at a high current density. However, in spite of significant improvements in its performance, the use of GaN-based LEDs is still seriously limited by the reduction in the external quantum efficiency (EQE) under high-injection-current-density conditions [8]. This well-known phenomenon is referred to as efficiency droop. Over the past years, several different suggestions regarding the mechanism of efficiency droop have been reported, including carrier leakage out of the multi-quantum well (MQW) active region [9, 10], Auger nonradiative recombination [11], junction heating [12], the dislocation density [13], non-uniform distribution of holes [14], polarization fields [15] and the quantum-confined Stark effect (QCSE) [16]. Nevertheless, the major origin of the efficiency droop behavior is still not completely understood. For this reason, many approaches have been suggested to increase EQE and mitigate efficiency droop in recent years. Among them, for the GaN-based blue LEDs, it is notable that not only the polarization field in MQWs but also low injection and transport of holes are believed to be the main factors of the efficiency droop and the degradation in EQE. Conventionally, the strong piezoelectric and spontaneous polarization due to large lattice mismatch between InGaN well and GaN barrier layers cause an internal electric field perpendicular to the plane of the well layer, resulting in poor overlap between electron and hole wavefunctions in the well [17]. These effects give rise to decreased internal quantum efficiency (IQE). Furthermore, a p-type AlGaN electron blocking layer (EBL) is usually used between the p-type $\mathrm{GaN}$ layer and the active region to alleviate the electron leakage and to improve the device efficiency [18, 19]. The EBL has played an important role in effectively confining electrons in the active region because the large conduction band offset between the last GaN barrier and the AlGaN layer is beneficial in preventing electron leakage out of quantum wells. However, it has been reported that a large polarization field in $\mathrm{AlGaN}$ EBL can reduce the effective barrier height for electrons [20]. On the other

\footnotetext{
*Corresponding author: tgkim1@korea.ac.kr

Color versions of one or more of the figures in this paper are available online.
} 
hand, the downward band-bending induced by the polarization field and the valence band offset at the interface of the last barrier of the MQWs and the AlGaN layer might act as a potential barrier for holes [21, 22]. Therefore, the polarization-matched quarternary AlGaInN and InGaN quantum barriers have been suggested to reduce the polarization field in the active region and have been experimentally demonstrated to enhance the light-output power and to reduce the efficiency droop of LEDs [23, 24]. It has been also reported that p-AlGaInN and p-AlInN EBL in LED structures can prevent carrier leakage over the $\operatorname{EBL}[25,26]$.

In this paper, in order to solve the above mentioned problem, we propose and investigate the effect of GaN/ graded-composition $\mathrm{AlGaInN/GaN}$ as quantum barriers in the active region on the performance of GaN-based vertical LED in comparison with those of conventional GaN quantum barriers. The inserted graded-composition AlGaInN barriers were used to reduce polarization effects between quantum well and barrier layer so that the carrier distribution and radiative recombination rate were improved, and thereby efficiency droop was alleviated. We use the Simulator of Light Emitters based on Nitride Semiconductors (SiLENSe) and the Spreading of Current in Light Emitting Diodes (SpecLED) programs to analyze enhancement of the device performance [27].

\section{DEVICE STRUCTURE AND PARAMETERS}

As shown in Fig. 1, the vertical LED structures for the numerical analysis were composed of a $2.5-\mu m$-thick n-type $\mathrm{GaN}$ (n-doping $=5 \times 10^{18} \mathrm{~cm}^{-3}$ ). The MQW active region consisted of five 3-nm-thick undoped $\operatorname{In}_{0.2} \mathrm{Ga}_{0.8} \mathrm{~N}$ QWs separated by 12 -nm-thick barrier layers with a $3 \times 10^{18} \mathrm{~cm}^{-3}$ donor $(\mathrm{Si})$ concentration. On top of the active region were a 20-nm-thick p-type $\mathrm{Al}_{0.15} \mathrm{Ga}_{0.85} \mathrm{~N}$ EBL and a $0.2-\mu \mathrm{m}$-thick p-GaN (p-doping $=1.2 \times 10^{18} \mathrm{~cm}^{-3}$ for both layers) cap layer. For a comparative study, two LED structures were assumed. The schematic diagrams of the LED structures with two different types of quantum barriers are shown in Fig. 1. In order to reduce the polarization effect of the

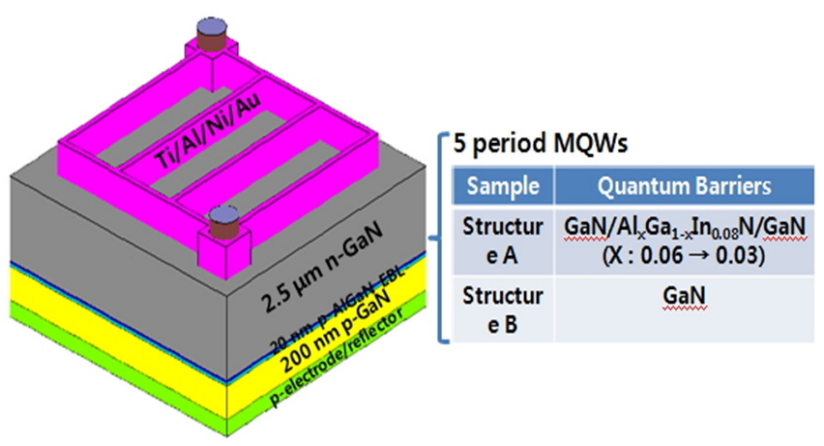

FIG. 1. Schematic diagrams of three GaN-based vertical LED structures of two different quantum barrier types. original LED structure with conventional GaN barriers, 8-nm-thick graded-composition AlGaInN layers are sandwiched by 2 -nm-thick $\mathrm{GaN}$ barriers and are proposed to replace the conventional GaN barrier. The Al composition (x) of the graded-composition $\mathrm{Al}_{\mathrm{X}} \mathrm{Ga}_{1-\mathrm{X}} \mathrm{In}_{0.08} \mathrm{~N}$ was gradually decreased from 0.06 to 0.03 . The VLED with conventional GaN barriers and that with $\mathrm{GaN} /$ graded-composition $\mathrm{AlGaInN} / \mathrm{GaN}$ barriers are referred to as structure $\mathrm{A}$ and structure $\mathrm{B}$, respectively. The band offset ratio, $\Delta \mathrm{E}_{\mathrm{C}} / \Delta \mathrm{E}_{\mathrm{V}}=0.7 / 0.3$, serves as a default parameter in the simulation. All the calculations are carried out with the threading dislocation density of $10^{8} \mathrm{~cm}^{-2}$. The operating temperature is assumed to be $300 \mathrm{~K}$. To simplify the simulation, the light extraction efficiency is assumed to be 0.7 . The electron and the hole mobilities were assumed to be $150 \mathrm{~cm}^{2} / \mathrm{Vs}$ and $10 \mathrm{~cm}^{2} / \mathrm{Vs}$, respectively. The area of the device geometry was $1 \times 1$ $\mathrm{mm}^{2}$. Other material parameters used in the simulation can be found in Ref. 28. For this simulation, the light-output power versus the current and the current versus the voltage (L-I-V) characteristics, the electroluminescence (EL) and internal quantum efficiency (IQE) were investigated by using the SimuLED package, which provides a coupled 3D analysis of the electrical, thermal, and optical processes.

The basic equations used for this study are Poisson's equation and the current-continuity equations for electrons and holes, as represented by the following equations [29]:

$$
\begin{aligned}
& \nabla \cdot(\varepsilon \nabla \psi)=-q\left(p-n+N_{D}-N_{A}\right) \\
& \frac{d n}{d t}=\frac{1}{q} \nabla \cdot J_{e}-R \\
& \frac{d p}{d t}=\frac{1}{q} \nabla \cdot J_{h}-R
\end{aligned}
$$

where $\psi$ is the electric potential, $\mathrm{n}$ is the electron density, $p$ is the hole density, $J_{e}$ and $J_{h}$ are the current densities for electrons and holes, respectively, and $\mathrm{R}$ is a recombination term. In Eq. (1), $\mathrm{N}_{\mathrm{D}}$ and $\mathrm{N}_{\mathrm{A}}$ represent the ionized donor concentration and ionized acceptor concentration, respectively. In the simulation, the recombination term $\mathrm{R}$ in Eqs. (2), (3) and (4) was represented as follows:

$$
R=R_{s p}+R_{S R H}+R_{A u g},
$$

where $R_{s p}, R_{S R H}$, and $R_{A u g}$ are the spontaneous emission recombination, the Shockley-Read-Hall nonradiative recombination, and the Auger recombination, respectively. Also, the internal quantum efficiency $\left(\eta_{\text {int }}\right)$ was given by

$$
\eta_{i}=\frac{\int R_{s p} d v}{\int R d v}
$$


The electron and the hole current densities are related to the fluxes as $\mathrm{j}_{\mathrm{n}}=-\mathrm{q} \cdot \mathrm{j}_{\mathrm{n}}$ and $\mathrm{j}_{\mathrm{p}}=+\mathrm{q} \cdot \mathrm{j}_{\mathrm{p}}$. In turn, the carrier fluxes depend generally on the gradients of the quasiFermi levels as

$$
J_{n}=-\frac{\mu_{n} n}{q} \nabla F_{n}, \quad J_{p}=-\frac{\mu_{p} p}{q} \nabla F_{p}
$$

where $\mu_{\mathrm{n}}$ and $\mu_{\mathrm{p}}$ are the electron and the hole mobilities, respectively. Moreover, commonly-accepted parameters are used in the simulations, including a Shockley-Read-Hall (SRH) recombination lifetime of $50 \mathrm{~ns}$ and an Auger recombination coefficient of $1 \times 10^{-31} \mathrm{~cm}^{6} \mathrm{~s}^{-1}$ [30] at the interfaces between the $\mathrm{InGaN}$ well and $\mathrm{GaN}$ barrier in the active region.

\section{RESULTS AND DISCUSSION}

Figure 2 shows the simulated the light-output power versus the current and the current versus the voltage (L-I-V) characteristics for the two LED structures of different quantum barrier types as functions of the injection current. The forward voltages at $350 \mathrm{~mA}$ of structure A and $\mathrm{B}$ are 4.0 and $3.5 \mathrm{~V}$, respectively. The reduced forward voltage can be attributed to the improvement in the hole injection and distribution by the reduced polarization field of graded-composition AlGaInN barriers. The light output powers of the two LEDs increases as the injection current increases. The output power of structure B was found to be higher than that of structure $A$ over the whole range of current. The light output power of structure B was higher by $4.3 \%$ than that of structure A. Also, the IQEs of structure $\mathrm{A}$ and $\mathrm{B}$ as functions of injection current density are shown in Fig. 3. The IQE of structure B was increased by $9.51 \%$ at an injection current of $350 \mathrm{~mA}$ compared with that of structure A. The efficiency droop defined as $\left(\eta_{\text {peak }}-\eta_{350 \mathrm{~A} / \mathrm{cm} 2}\right) / \eta_{\text {peak }}$, is alleviated from $24.8 \%$ to $13.3 \%$, indicating the improvement of radiative-recombination rate

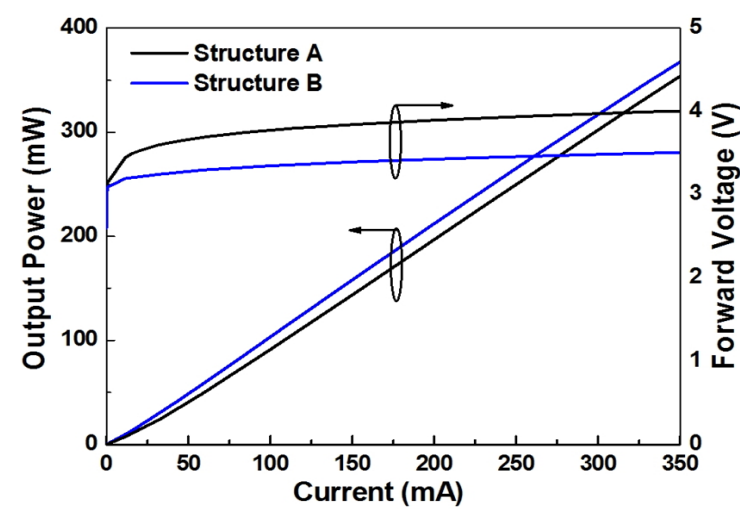

FIG. 2. L-I-V curves of LEDs with two different quantum barrier types. due to efficient hole injection and distribution. These results indicate that the structure $\mathrm{B}$ is the superior one due to the reduced efficiency droop and higher light output power at high current. The cause for the superiority of structure B is the combined effect of the efficient injection and uniform distribution of hole and the enhanced radiative-recombination rates.

In order to explain the above phenomenon, the calculated energy band diagrams and quasi-Fermi levels of structure $\mathrm{A}$ and $\mathrm{B}$ at an injection current of $35 \mathrm{~A} / \mathrm{cm}^{2}$ are shown in Fig. 4. In Fig. 4(a), the LED with conventional GaN barriers

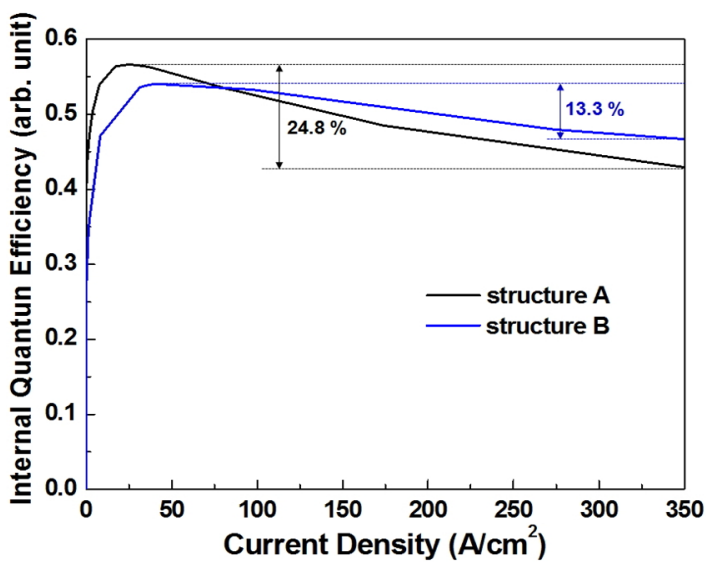

FIG. 3. IQE of LEDs with structure A and B as functions of injection current.
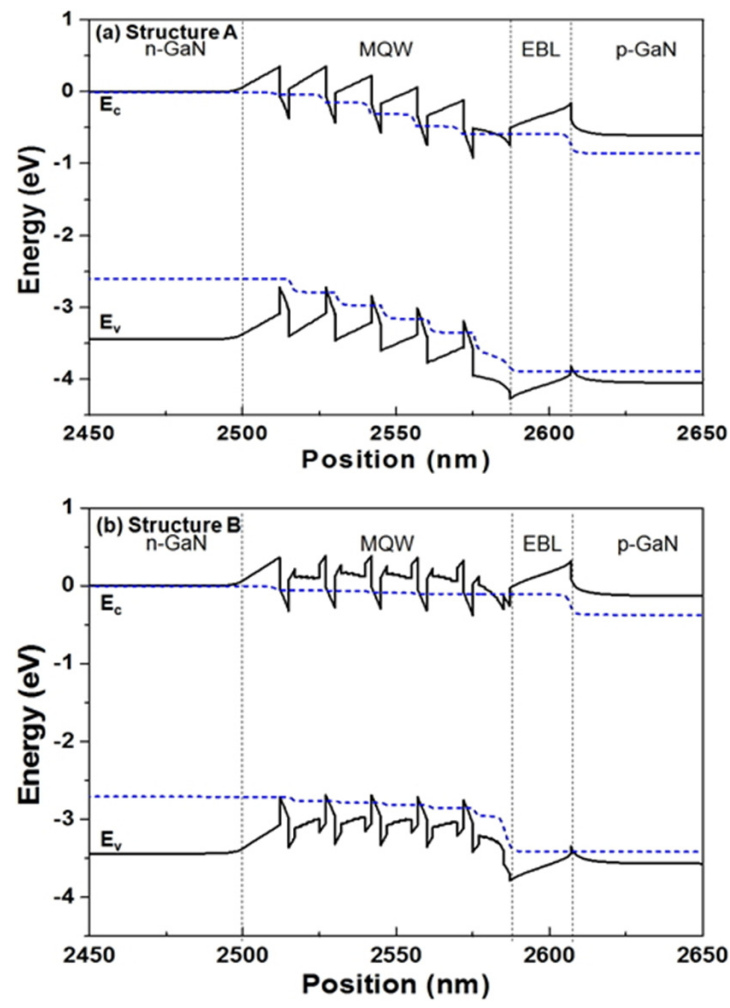

FIG. 4. Calculated band diagrams of LEDs with (a) structure $\mathrm{A}$ and (b) structure $\mathrm{B}$ at $35 \mathrm{~A} / \mathrm{cm}^{2}$. 
show highly tilted energy band structures in the active regions. This means that the polarization induced internal field causes the increase of the potential difference in the energy band between the well and barrier layers, and thus the reduction of radiative recombination rate and IQE is occurred by poor overlap between the electron and hole wave functions. In addition, the increased potential height in valence band prevents efficient hole transport from the p-type GaN into active regions. On the other hand, in Fig. 4(b), because of the relative small band bending induced by the
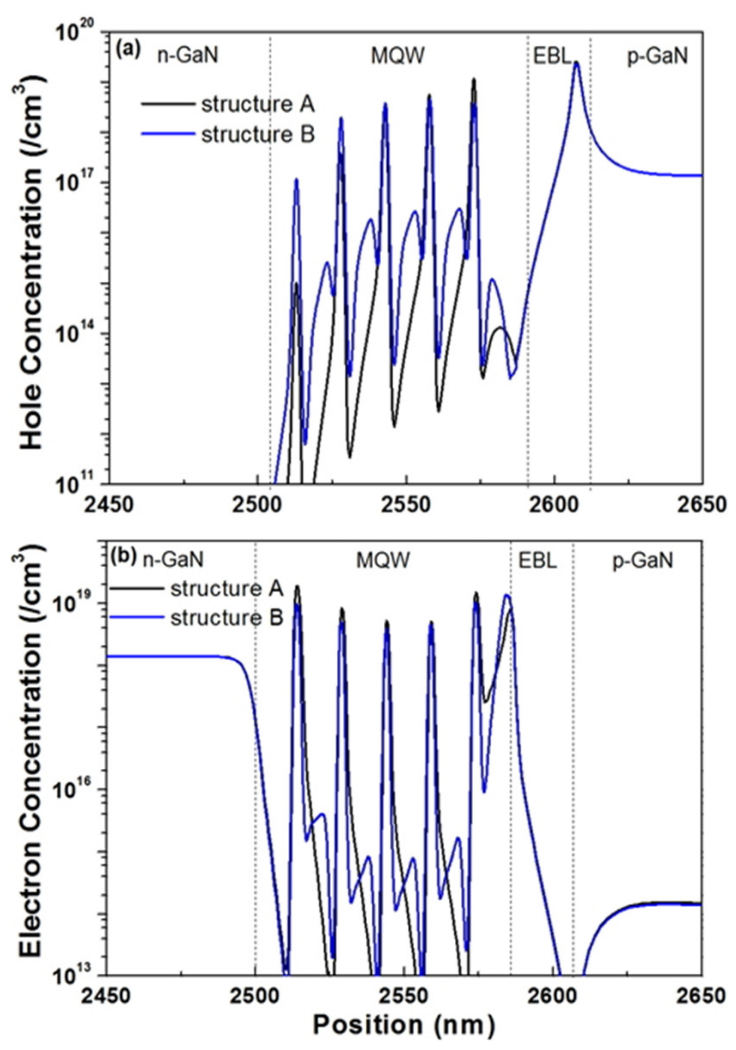

FIG. 5. (a) Hole concentrations and (b) electron concentrations of LEDs with two different quantum barrier types at $35 \mathrm{~A} / \mathrm{cm}^{2}$.

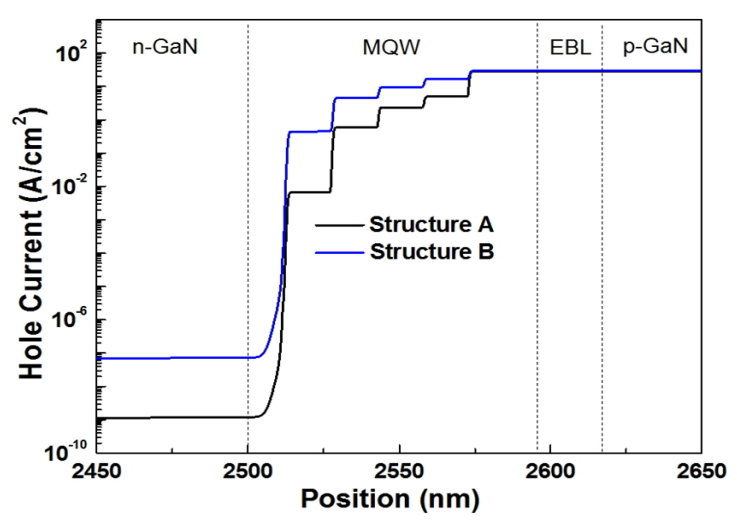

FIG. 6. Simulated hole current densities close to of the active regions of LEDs with two different quantum barrier types at $35 \mathrm{~A} / \mathrm{cm}^{2}$. reduced internal field in active regions, the slope of valence band in the inserted graded-composition AlGaInN barriers is alleviated. Therefore, these barriers of structure $B$ can attribute to a decreased effective potential barrier height for the injection and distribution of holes without the leak of electrons out of the active regions.

To explain the above-mentioned reasons, the electron and hole concentration of the structure $\mathrm{A}$ and $\mathrm{B}$ at 35 $\mathrm{A} / \mathrm{cm}^{2}$ are shown in Fig. 5. For the structure B, the hole concentration decreased at fifth quantum well near the $\mathrm{p}-\mathrm{GaN}$ side. However, at the same time, the hole concentrations of first, second and third quantum wells is nearly one or two order of magnitude higher than that of the structure A, which is shown in Fig. 5(a). Simultaneously, electrons are uniformly distributed throughout the entire MQW regions in the structure A and B, as shown in Fig. 5(b). A comparison of the hole concentrations in the structure $\mathrm{A}$ and $\mathrm{B}$ around the active regions shows a uniform hole distribution for the structure $B$ because the GaN/gradedcomposition AlGaInN/GaN barriers more effectively transport holes. In other words, larger numbers of holes inside the active regions, as well as enhanced overlap of the electron and the hole wave functions, result in the increased radiative recombination. These improved hole injection and distribution results can be further explained by the hole current inside the MQWs.

Figure 6 shows the hole injection current densities as functions of position close to the active regions of the LEDs with two different quantum barrier types at $35 \mathrm{~A} / \mathrm{cm}^{2}$. The hole current density of the structure $\mathrm{B}$ injected into the active region from the $\mathrm{p}-\mathrm{GaN}$ is higher than that of the structure A. Thus, it is clearly apparent that the GaN/gradedcomposition AlGaInN/GaN barriers enable more efficient hole transport across the MQWs, which is beneficial to the high output power and the reduction of efficiency droop.

\section{CONCLUSION}

In conclusion, we numerically studied the influence of GaN/graded-composition AlGaInN/GaN quantum barriers in the active region on the performance of GaN-based VLEDs. The simulation results showed that, among the two LED structures under study, the LED with GaN/gradedcomposition AlGaInN/GaN quantum barriers revealed the enhanced electrical and optical characteristics. The forward voltages at $350 \mathrm{~mA}$ were calculated to be 3.5 and $4.0 \mathrm{~V}$ for VLEDs with GaN/graded-composition AlGaInN/GaN barriers and GaN barriers, respectively. The output power and internal quantum efficiency of LEDs with GaN/graded-composition $\mathrm{AlGaInN} / \mathrm{GaN}$ barriers was higher by $4.3 \%$ and $9.51 \%$ than those of LEDs with conventional GaN barriers, respectively. The efficiency droop of LEDs using GaN/graded-composition $\mathrm{AlGaInN} / \mathrm{GaN}$ barriers was alleviated from $24.8 \%$ to $13.3 \%$, indicating the improvement of radiative-recombination rate, in comparison with that of LEDs using GaN barriers. Such 
enhancements could be attributed to the relatively efficient hole injection and uniform distribution in MQWs due to the moderate downward band bending and the high radiative recombination rate in the active regions, and the small efficiency droop. This proposed quantum barrier structure within LEDs is beneficial, especially for GaN-based LEDs operating at high injection current.

\section{ACKNOWLEDGMENT}

This work was supported by the National Research Foundation of Korea (NRF) grant funded by the Korea government (No. 2011-0028769).

\section{REFERENCES}

1. F. F. Jobsis, M. R. Krames, O. B. Shchekin, R. MuellerMach, G. O. Mueller, L. Zhou, G. Harbers, and M. G. Craford, "Status and future of high-power light-emitting diodes for solid-state lighting," IEEE J. Display Technol. 3, 160-175 (2007).

2. D. J. Chae, D. Y. Kim, D. H. Kim, S. J. Kim, and T. G. Kim, "Optical properties of $\mathrm{NiO} / \mathrm{Al}$ based reflector for high-power ultraviolet light-emitting diodes," J. Korean Phys. Soc. 58, 990-993 (2011).

3. J. W. Yang, J. I. Sim, H. M. An, and T. G. Kim, "Fabrication of nanometer-scale pillar structures using nanosphere lithography," J. Korean Phys. Soc. 58, 994-997 (2011).

4. Y. C. Shin, D. H. Kim, E. H. Kim, J. M. Park, K. M. Ho, K. Constant, J. H. Choe Park, Q. H. Park, H. Y. Ryu, J. H. Baek, T. Jung, and T. G. Kim, "High efficiency GaN light-emitting diodes with two dimensional photonic crystal structures of deep-hole square lattices," IEEE J. Quantum Electron. 46, 116-120 (2010).

5. S. J. Kim, T. Y. Nam, and T. G. Kim, "Low-resistance nonalloyed Ti/Al ohmic contacts to N-face n-type GaN with $\mathrm{O}_{2}$ plasma treatment," IEEE Electron Device Lett. 32, 149-151 (2011).

6. D. H. Kim, S. J. Kim, S. H. Kim, T. Jeong, S.-M. Hwang, and T. G. Kim, "Improved device performance in nonpolar a-plane GaN LEDs using an $\mathrm{Ni} / \mathrm{Al} / \mathrm{Ni} / \mathrm{Au}$ n-type ohmic contact," Phys. Stat. Sol. (RRL) 5, 274-276 (2011).

7. C. G. Son, J. H. Yi, J. S. Gwag, J. H. Kwon, and G. J. Park, "Improvement of color and luminance uniformity of the edge-lit backlight using the RGB LEDs," J. Opt. Soc. Korea 15, 272-277 (2011).

8. Y.-L. Li, Y.-R. Huang, and Y.-H. Lai, "Efficiency droop behaviors of $\mathrm{InGaN} / \mathrm{GaN}$ multiple-quantum-well light-emitting diodes with varying quantum well thickness," Appl. Phys. Lett. 91, 181113 (2007).

9. L.-B. Chang, M.-J. Lai, R.-M. Lin, and C.-H. Huang, "Effect of electron leakage on efficiency droop in wide-well InGaN-based light-emitting diodes," Appl. Phys. Express 4, 012106 (2011).

10. K.-S. Kim and J. C. Yi, "Epitaxial structure optimization for high brightness InGaN light emitting diodes by using a self-consistent finite element method," J. Opt. Soc. Korea 16, 292-298 (2012).

11. Y. C. Shen, G. O. Mueller, S. Watanabe, N. F. Gardner, A. Munkholm, and M. R. Krames, "Auger recombination in InGaN measured by photoluminescence," Appl. Phys. Lett. 91, 141101 (2007).

12. A. A. Efremov, N. I. Bochkareva, R. I. Gorbunov, D. A. Lavrinovich, Y. T. Rebane, D. V. Tarkhin, and Y. G. Shreter, "Effect of the joule heating on the quantum efficiency and choice of thermal conditions for high-power blue InGaN/GaN LEDs," Semiconductors 40, 605-610 (2006).

13. M. F. Schubert, S. Chhajed, J. K. Kim, and E. F. Schubert, D. D. Koleske, M. H. Crawford, S. R. Lee, A. J. Fischer, R. G. Thale, and M. A. Banas, "Effect of dislocation density on efficiency droop in GaInN/GaN light-emitting diodes," Appl. Phys. Lett. 91, 231114 (2007)

14. A. David, M. J. Grundmann, J. F. Kaeding, N. F. Gardner, T. G. Mihopoulos, and M. R. Krames, "Carrier distribution in (0001) InGaN/GaN multiple quantum well light-emitting diodes," Appl. Phys. Lett. 92, 053502 (2008).

15. M. H. Kim, M. F. Schubert, Q. Dai, J. K. Kim, E. F. Schubert, J. Piprek, and Y. Park, "Origin of efficiency droop in GaN-based light-emitting diodes," Appl. Phys. Lett. 91, 183507 (2007).

16. J. S. Cabalu, C. Thomidis, T. D. Moustakas, S. Riyopoulos, L. Zhou, and D. J. Smith, "Enhanced internal quantum efficiency and light extraction efficiency from textured GaN/AlGaN quantum wells grown by molecular beam epitaxy," J. Appl. Phys. 99, 064904 (2006).

17. S.-H. Park and S.-L. Chuang, "Piezoelectric effects on electrical and optical properties of wurtzite GaN/AlGaN quantum well lasers," Appl. Phys. Lett. 72, 3103 (1998).

18. A. Chitnis, J. P. Zhang, V. Adivarahan, M. Shatalov, S. $\mathrm{Wu}$, R. Pachipulusu, V. Mandavilli, and M. A. Khan, "Improved performance of 325-nm emission AlGaN ultraviolet light-emitting diodes," Appl. Phys. Lett. 82, 2565 (2003).

19. R. C. Tu, C. J. Tun, S. M. Pan, C. C. Chuo, J. K. Sheu, C. E. Tsai, and G. C. Chi, "Improvement of nearultraviolet InGaN-GaN light-emitting diodes with an AlGaN electron-blocking layer grown at low temperature," IEEE Photon. Technol. Lett. 15, 1342-1344 (2003).

20. L. Zhang, K. Ding, N. X. Liu, T. B. Wei, X. L. Ji, P. Ma, J. C. Yan, J. X. Wang, Y. P. Zeng, and J. M. Li, "Theoretical study of polarization-doped GaN-based light-emitting diodes," Appl. Phys. Lett. 98, 101110 (2010).

21. Y. K. Kuo, J. Y. Chang, and M. C. Tsai, "Enhancement in hole-injection efficiency of blue InGaN light-emitting diodes from reduced polarization by some specific designs for the electron blocking layer," Opt. Lett. 35, 3285-3287 (2010).

22. S. H. Yen, M. C. Tsai, M. L. Tsai, Y. J. Shen, T. C. Hsu, and Y. K. Kuo, "Effect of N-type AlGaN layer on carrier transportation and efficiency droop of blue InGaN light-emitting diodes," IEEE Photon. Technol. Lett. 21, 975-977 (2009).

23. Y.-K. Kuo, J.-Y. Chang, M.-C. Tsai, and S.-H. Yen, "Advantages of blue InGaN multiple-quantum light-emitting diodes with InGaN barriers," Appl. Phys. Lett. 95, 011116 (2009).

24. Y.-K. Fu, R.-H. Jiang, Y.-H. Lu, B.-C. Chen, R. Xuan, Y.-H. Fang, C.-F. Lin, Y.-K. Su, and J.-F. Chen, "The effect of trimethylgallium flows in the AlInGaN barrier on 
Numerical Study of Enhanced Performance in InGaN Light-Emitting Diodes with $\cdots$ - Su Jin Kim and Tae Geun Kim 21

optoelectronic characteristics of near ultraviolet light-emitting diodes grown by atmospheric pressure metalorganic vapor phase epitaxy," Appl. Phys. Lett. 98, 121115 (2011).

25. S. Choi, H. J. Kim, S. S. Kim, J. Liu, J. Kim, J. H. Ryou, R. D. Dupuis, A. M. Fischer, and F. A. Ponce, "Improvement of peak quantum efficiency and efficiency droop in III-nitride visible light-emitting diodes with an InAlN electron-blocking layer," Appl. Phys. Lett. 96, 221105 (2010).

26. Y. K. Kuo, M. C. Tsai, and S. H. Yen, "Numerical simulation of blue InGaN light-emitting diodes with polarizationmatched AlGaInN electron-blocking layer and barrier layer," Opt. Commun. 282, 4252-4255 (2009).
27. SiLENSe and SpeCLED by STR Group Inc., St.-Petersburg, Russia, http://www.str-soft.com.

28. I. Vurgaftman and J. R. Meyer, "Band parameters for nitrogen-containing semiconductors," J. Appl. Phys. 94, 3675 (2003).

29. M. Kurata, Numerical Analysis for Semiconductor Devices (D. C. Heath \& Co., Lexington, MA, USA, 1982).

30. Y. C. Shen, G. O. Müeller, S. Watanabe, N. F. Gardner, A. Munkholm, and M. R. Krames, "Auger recombination in InGaN measured by photoluminescence," Appl. Phys. Lett. 91, 141101 (2007). 